\title{
Antimicrobial susceptibility testing and the Clinician.
}

The clinicians are confronted with infections in their daily practice, and most of the times they rely on national or international data available to treat these infections. Sometimes it works but sometimes organisms do not respond to antimicrobials due to a variety of factors.

The data clinician applies is most of the time extrapolated from higher centers, centers of excellence in infectious diseases or he follows guidelines issued by various reputed organizations like WHO, CDC Atlanta, IDSA, NICE, etc.

Algorithms followed by the medical fraternity in managing infections have been changing with the advent of automation or computerization. Infectious disease diagnostics and management too has been changing fast since the advent of antibiotics. At the same time, these precious molecules are being used inadvertently due to the lack of isolation of organisms.

A few years back clinicians were perplexed with conflicting outcomes of various microbiologic results from different medical centers that used to rely on age-old diagnostics modalities in microbiology.

One of the landmarks in modern medicine is the determination of susceptibility of a particular pathogen to select an antimicrobial drug with its adequate blood concentration. In vitro susceptibility of the organism to antibiotics remains a Herculean task. These tests need to be properly sampled, stored, incubated, reproduced and accurately performed despite edgy clinician who intends to treat the infection on a war footing.

The clinicians are confronted with infections in their daily practice, and most of the times they rely on national or international data available to treat these infections.

\section{Access this article online}

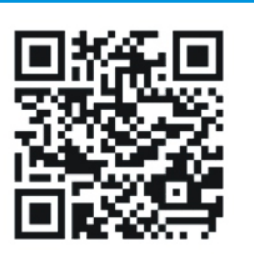

Website:

www.jmsskims.org

DOI:

https://doi.org/10.33883/jms.v22i2.499
Algorithms followed by the medical fraternity in managing infections have been changing with the advent of automation or computerization. Infectious disease diagnostics and management too has been changing fast since the advent of antibiotics. At the same time, these precious molecules are being used inadvertently due to the lack of isolation of organisms.

A few years back clinicians were perplexed with conflicting outcomes of various microbiologic results from different medical centers that used to rely on age-old diagnostics modalities in microbiology.

One of the landmarks in modern medicine is the determination of susceptibility of a particular pathogen to select an antimicrobial drug with its adequate blood concentration. In vitro susceptibility of the organism to antibiotics remains a Herculean task. These tests need to properly sampled, stored, incubated, reproduced and accurately performed despite edgy clinician who intends to treat the infection on a war footing.

The microbial tests are performed in laboratories guided by CLSI guidelines. These guidelines are annually published, reviewed by clinical and laboratory standard institute, formerly called as National Committee for Clinical Laboratory Standards (NCCLS). This standard group is having a representation of clinical groups, laboratory, industry, and governmental agencies.

A number of tests are employed for finding antimicrobial sensitivity of microbes ranging from dilution methods, diffusion methods, quantitative methods, qualitative methods, and manual, automated, conventional to rapid methods.

Every method employed has its own dimension but present scenario in era of artificial intelligence microbial detection

How to cite this article: Koul AN. Antimicrobial susceptibility testing and the Clinician. jms 2019;22(2):1-2

Received: 14-12-2019 Accepted: 14-12-2019 
Koul AN; Antimicrobial susceptibility testing and the Clinician.

and sensitivity has been revolutionized by automated systems (Bactec, Vitek 2).

Infections are a very important cause of morbidity and mortality worldwide and prompt diagnosis with proper management in the desired methodology to improvise the outcomes in such patients.

In this context, the authors here have tried to compare conventional methods of incubation to automated systems for picking up infecting organisms as well as predicting antimicrobial sensitivity.

Though the authors have confined themselves to gram positive organisms such a study may be extended to gramnegative infections. It is pertinent to mention here the dispersion of gram-negative microbes and consequences thereof are of enormous magnitude in India as compared to western countries.

The average incubation time of automated has improved over conventional methods. It has been regarded that despite prior antibiotic usage the predictive value of cultivation of organisms may be better with automated systems with a caveat of higher false positivity, especially of commensals. It is presumed that the presence of nonionic adsorbent resins remove antibiotics from drawn blood to neutralize their impact on cultivating organisms.

As mentioned earlier every system has a flaw, the abovementioned study needs to cater to drawbacks like some of the organisms depending on their respective metabolism. Organisms may not be effectively detected by an automated system or maybe wrongly specified or specie typed in automation. Here come the role of microbiologist to separate such colonies with manual or semi automated analysis.

It is heartening to find in this study that no major errors were found in the isolates studied. Though few errors within CLSI norms were found for enterococus to vancomycin and ampicillin.

I second the authors to be advised that testing with manual method for proper selection of antibiotic.
I do acknowledge that many factors like patient factors, sampling factors, institutional background, academic programme, financial implications, adherence to CLSI guidelines, accreditation of microbial testing do have a lot of bearing on outcomes of blood cultures with subsequent antimicrobial usage and ultimately impact on the infection limitation.

With evidence-based, targeted therapy becoming a norm in modern medicine and infections need to be dealt with on high priority note. Such a study needs to be replicated to all organisms in adult as well as paediatric infectious diseases with a caveat that in vitro and in vivo susceptibility may be still a point of concern without undermining importance of testing.

\section{REFERENCES}

1. Surase PV, Nataraj G, Pattamadai K etal. An appropriately performed conventional blood culture can facilitate choice of therapy in resource constrained settings comparison with BACTEC 9050. J Postgrad Med.2016, 62:228-34.

2. Performance standards for antimicrobial susceptibility testing. 26 Ed. supplement M100S. Wayne PA. Clinical and laboratory standards institute: 2016.pp1-82.

3. Bose S, Vishal G. Utility of BACTEC Blood Culture System versus Conventional Blood Culture Method for Detection of Bacteriaemia in Pediatric Patients. International Journal of Current Microbiology and Applied Sciences. 2018 Oct 20; 7(10):112631.

Dr. Ajaz Nabi Koul.

MD, MRCP (UK), MRCP (London), FCAN (SGRH

Delhi), FRCP (Edinburgh)

Additional Professor.

Department of Medicine \& Infectious Diseases,

SKIMS, SGR. 